\title{
A comparative study between Plasma vitamin c level and severity of knee osteoarthritis
}

\author{
Dr. Subhrajyoti Naskar ${ }^{1}$, Dr. Indranil Dawn ${ }^{2}$, Dr Susmita Sarkar ${ }^{3}$, Dr.Chinmoy \\ $\mathrm{De}^{4}$, Dr. Gouranga Biswas ${ }^{5}$. \\ ${ }^{1}$ Faculty member of Community Medicine, Malda Medical College,Malda,india \\ ${ }^{2}$ Faculty members of Biochemistry, Malda Medical College,Malda \\ ${ }^{3}$ Faculty member of Biochemistry, Malda Medical College,Malda,india \\ ${ }^{4}$ Faculty Member of Orthopaedics, Burdwan Medical College,Burdwan \\ ${ }^{5}$ Faculty Member of paediatrics,Malda Medical College,Malda
}

\begin{abstract}
Most prominent feature in osteoarthritis is the progressive destruction of articular cartilage which results in impaired joint motion, severe pain, structural and functional failure of synovial joints. A hospital based cross sectional study was conducted in Burdwan Medical College, West Bengal, India on 76 postmenopausal women aged 45-70 years suffering from Osteoarthritis (OA) of the knee joint, which was diagnosed from symptoms, clinical examinations and radiographic findings. 150 subjects undergoing knee joint arthroscopy for chronic knee pain, meniscal tears or anterior cruciate ligament reconstruction were taken as control plasma vitamin $C$ was measured. The width of knee joint space was measured from the radiographic plates to assess the disease severity. Knee joint radiographs were evaluated with the Kellgren-Lawrence grading scale.Furthermore both were correlated with plasma vitamin $C$ levels to find out possible association between the vitamin $C$ and the disease progression. Results showed that there was a significant decrease in vitamin C levels in patients with knee joint osteoarthritis as compared to the controls. Plasma vitamin C showed negative correlation with Kellgren-Lawrence grading and positive correlation with knee joint space width. Knee joint space width and vitamin C decreased with increasing Kellgren-Lawrence grade.
\end{abstract}

Keywords- vitamin C.Knee Osteoarthritis, Kellgren-Lawrence grading scale,

\section{Introduction}

Osteoarthritis (OA, also known as degenerative arthritis, degenerative joint disease), is a clinical syndrome in which low-grade inflammation results in pain in the joints. Osteoarthritis of the knee is a major cause of mobility impairment, particularly among females. Its most prominent feature is the progressive destruction of articular cartilage which results in impaired joint motion, severe pain, structural and functional failure of synovial joints ${ }^{1}$.

The two major macromolecules in cartilage are type 2 collagen, which provides cartilage its tensile strength, and aggrecan, a proteoglycan macromolecule linked with hyaluronic acid, which consists of highly negatively charged glycosaminoglycans. In normal cartilage, type 2 collagen is woven tightly, constraining the aggrecan molecules in the interstices between collagen strands, forcing these highly negatively charged molecules into close proximity with one another. The aggrecan molecule, through electrostatic repulsion of its negative charges, gives cartilage its compressive stiffness. Cartilage matrix synthesis and catabolism are in a dynamic equilibrium influenced by the cytokine and growth factor environment and by mechanical stress. While healthy cartilage is metabolically sluggish, with slow matrix turnover and a net balance of synthesis and degradation, cartilage in early OA or after an injury is highly metabolically active. OA cartilage is characterized by gradual depletion of aggrecan, an unfurling of the tightly woven collagen matrix, and loss of type 2 collagen. With these changes comes increasing vulnerability of cartilage, which no longer has compressive stiffness. ${ }^{2}$

Vitamin C (ascorbic acid) is an important water soluble vitamin in biological fluids and an essential micronutrient required for normal metabolic functioning of the body ${ }^{3}$ Ascorbic acid (Asc) is required for the synthesis of the most abundant protein in cartilage, type II collagen. Ascorbate and ascorbic acid increased protein and proteoglycan synthesis by articular chondrocytes ${ }^{4-6}$ and increased the mRNA levels of type I and II collagen ${ }^{7,8}$ and aggrecan and $\alpha$ - prolyl 4-hydroxylase ${ }^{9}$. It decreased the lipopolysaccharide (LPS)-induced GAG release $^{\mathbf{1 0}}$. It also affected the activities of lysosomal enzymes, decreasing the activities of arylsulfatase A and arylsulfatase $\mathrm{B}$, an $\mathrm{N}$ - acetylgalactosaminidase-4-sulfatase (prevent aggrecan damage).

However, few studies have been done to establish the relationship between oxidative stress and clinical severity of disease. Aim of this study was to analyze the relationship between plasma vitamin c level and the clinical severity of osteoarthritis. The clinical severity of OA was evaluated by measuring the width of knee joint space and by utilizing the Kellgren-Lawrence grading scale. The width of knee joint space was measured 
from the radiographs of the same. The severity of OA was graded by the Kellgren-Lawrence grading scale

${ }^{11}$ which is as follows:

Grade 1: doubtful narrowing of joint space and possible osteophytic lipping;

Grade 2: definite osteophytes and possible narrowing of joint space;

Grade 3: moderate multiple osteophytes, definite narrowing of joints space, some sclerosis and possible deformity of bone contour;

4: large osteophytes marked narrowing of joint space, severe sclerosis and definite deformity Grade of bone contour.

\section{Methodology}

The present hospital based case control study was undertaken in the Department of Biochemistry with collaboration of the Department of Orthopedic, Burdwan Medical College and Hospital, West Bengal. The study was conducted during the period from January 2008 to February 2009. The cases were selected from the patients attending the outdoor of Orthopedic Department of Burdwan Medical College and Hospital.

\subsection{Subjects}

The present study is based on 76 postmenopausal women aged 45-70 years (mean of 54.5) with OA of the knee, which was diagnosed from clinical symptoms, examinations and radiographic findings. Secondary OA patients, such as post-traumatic OA cases, were excluded from the study. 150 subjects were taken as control. All patients fulfilled the ACR criteria for knee OA ${ }^{12}$ The procedures followed were in accordance with the principles of the Declaration of Helsinki in 1975, as revised in 1983.

\subsection{Knee joint radiographs}

Antero-posterior weight-bearing radiographs of both knees were taken. The bilateral weight-bearing antero-posterior knee radiograph was taken with the patient standing with toes pointed straight ahead, knees fully extended, and weight equally distributed on both feet. The Kellgren-Lawrence grading of radiographs was done by a radiologist who was blinded to the source of subjects. The joint space width of the medial and lateral compartments of the knee joint was measured in millimeters. A vertical line was drawn from the midfemoral medial and lateral condyles to the tibial plateau, and the lesser of the two measurements was taken as the joint space width ${ }^{13}$

\subsection{Determination of plasma ascorbic acid (Photometric method)}

Ascorbic acid in plasma is oxidised by $\mathrm{Cu}^{2+}$ to form dehydroascorbic acid, which reacts with 2,4 dinitrophenylhydrazine to form a red bis-hydrazone, which is measured at $520 \mathrm{~nm}^{14}$.

\subsection{Specimen}

Heparinized blood was collected and centrifuged to obtain the plasma

\section{Result}

The obtained data was analyzed using SPSS software.

The Table 1 displays the comparison of mean of plasma vitamin $\mathrm{c}$ in cases and controls. The analysis of the different between the means was done with independent sample $t$ test. Plasma vitamin $\mathrm{c}$ was found to be significantly lower in the cases compared to the control. In the Table 2 the data of the knee joint space width and plasma vitamin $\mathrm{c}$ of the entire subject categorized as per the Kellgren-Lawrence grading scale. It is evident from the data that the mean of the knee joint space width and vitamin $\mathrm{c}$ are diminishing with increasing grading of knee joint osteo arthritis. Analysis of correlation between knee joint space width and Kellgren-Lawrence grading-was done by Pearson's correlation (Table 3). It was found that the decrease in the knee joint space width was significant. ( $\mathrm{p}$ value $<0.001$ ). ANOVA of the knee joint space width and plasma vitamin $\mathrm{c}$ of the various groups of subjects categorized clinically as per the Kellgren -Lawrence grading scale shows there is significant difference between them.(Table 4). From the table 5 it is evident that plasma vitamin c has a positive correlation with knee joint space width and negative correlation with radiological grading. The relationship between the Kellgren-Lawrence grading and the plasma vitamin $\mathrm{c}$ is shown in box whisker plot (Fig 1). Here the plasma vitamin c decreases with increasing Kellgren-Lawrence grade. Plasma vitamin C showed variances of 0.011 , $0.006,0.002,0.002$ and skewness of $0.603,0.907,-.054,-0.463$ for grade 1 , grade 2 , grade 3 and grade 4 respectively. 


\section{Discussion}

The present study was undertaken to analyze the association between plasma vitamin $\mathrm{C}$ and severity of knee osteoarthritis. We studied two radiographic grading criteria: Kellgren-Lawrence grading and joint space width. These two methods for evaluating the degree of OA are widely used. In the present study they were significantly related to each other: the Kellgren-Lawrence grade varied inversely with joint space width. (table 2) Pearson's bivariate correlation was done between knee joint space width and Kellgren-Lawrence grading, which showed a significant strong negative correlation (Table 3).

Plasma vitamin c levels, has decrease significantly in patients with knee osteoarthritis. (Table 1, 2 and 4) Plasma vitamin $\mathrm{c}$ also showed a significant strong positive correlation with knee joint space width and negative correlation with radiological grading (Table 5). ANOVA of plasma vitamin c of the various groups of subjects categorized clinically as per the Kellgren -Lawrence grading scale shows there is significant difference between them (table 4). Plasma vitamin C was also decrease with increasing Kellgren-Lawrence grade. (Box whisker plot-fig-1)

All data signified that with decreasing vitamin $\mathrm{c}$ level there was simultaneous increase severity of knee osteoarthritis. Schwartz and Adamy reported a decreased level of active proteinase in the presence of ascorbic acid and found further that sulphated proteoglycan biosynthesis; a presumed measure of repair was significantly increased in the cartilage in the presence of ascorbic acid. Manson et al suggested ascorbic acid stimulates collagen synthesis and modestly stimulates synthesis of aggrecan (a proteoglycan present in articular cartilage). Framingham epidemiological study found a threefold reduction in risk of OA progression for both the middle and highest tertiles of Vit $\mathrm{C}$ intake and an inverse association between Vit $\mathrm{C}$ intake and cartilage loss ${ }^{15}$. Schwartz and Leveille in two studies showed that high vitamin C (which would correspond to vitamin $\mathrm{C}$ in humans of at least $500 \mathrm{mg} /$ day) is helpful in preventing cartilage fibrillation, structural changes in the joint and eburnation in guinea pigs ${ }^{16}$

So reduce level of plasma vitamin C may responsible for worsening knee osteoarthritis.

\section{Conclusion}

The present study conducted an analysis on estimation of plasma vitamin $\mathrm{C}$ in the knee osteoarthritis patient's and how it related with disease severity. Plasma vitamin C level was significantly lower in the osteoarthritis patients as compared with the control population. The study, hence, suggests that treatment with vitamin $\mathrm{C}$ in the initial stages of the disease may be useful as secondary therapy to prevent the cartilage damage and deterioration of the musculoskeletal tissues in osteoarthritis. But further multicentre placebo controlled trials are needed to establish it. There was a significant positive correlation between the plasma vitamin $\mathrm{c}$ level and the severity of the disease process as indicated by the knee joint space width and radiological grading. Plasma vitamin $\mathrm{C}$ could be used as a marker to assess the disease severity of osteoarthritis. plasma could be used to complement knee joint space width.

\section{Reference}

[1] Yelin E. The economics of osteoarthritis. In Osteoarthritis. Oxford University Press; Oxford: 2003:17-21.

[2] Kenneth D. Brandt., osteoarthritis, Harrison principle of medicine 17 edition ;pages 2032-2045

[3] Shils, ME, Olson JA, Shiike M. Eds.: Modern Nutrition in Health in Disease. $8^{\text {th }}$ ed. Philadelphia, Lea \& Febiger, 1994

[4] Daniel JC, Pauli BU, Kuettner KE. Synthesis of cartilage matrix by mammalian chondrocytes in vitro. III. Effects ofascorbate. $J$ Cell Biol. 1984 Dec; 99(6):1960-9.

[5] Sandell LJ, Daniel JC. Effects of ascorbic acid on collagen mRNA levels in short term chondrocyte cultures. Connect Tissue Res. 1988; 17(1):11-22.

[6] Tiku ML, Gupta S, Deshmukh DR. Aggrecan degradation in chondrocytes is mediated by reactive oxygen species and protected by antioxidants. Free Radic Res. 1999 May; 30(5):395-405

[7] McNulty AL, Vail TP, Kraus VB. Chondrocyte transport and concentration ofascorbic acid is mediated by SVCT2.Biochim Biophys Acta. 2005Jun;1712(2):212-21.

[8] Shikhman AR, Brinson DC, Valbracht J, etal. Cytokine regulation of facilitated glucose transport in human articular chondrocytes. JImmunol. 2001

[9] Cartilage: a possible mechanism throughwhich age is a risk factor for osteoarthritis.Arthritis Rheum. 2002 Jan; 46(1):14-23.

[10] Richardson S, Neama G, Phillips T, et al.Molecular characterization and partial cDNA cloning of facilitative glucosetransporters expressed in human articularchondrocytes; stimulation of 2-deoxyglucose uptake by IGF-1 and elevatedMMP-2 secretion by glucose deprivation.Osteoarthritis Cartilage. 2003Feb; 11(2):92-101.

[11] Spector TD, Cooper C. Radiographic assessment of osteoarthritis in population studies: whither Kellgren and Lawrence? Osteoarthritis Cartilage. 1993 Oct;1(4):203-206.

[12] Altman R, Asch E, Bloch D, Bole G, Borenstein D, Brandt K, et al. Development of criteria for the classification and reporting of osteoarthritis: classification of osteoarthritis of the knee. Arthritis Rheum 1986;29:1039-49.

[13] Masaaki Takahashi, Kenichi Naito, Masashi Abe, Tomokazu Sawada and Akira Nagano. Relationship between radiographic grading of osteoarthritis and the biochemical markers for arthritis in knee osteoarthritis; 2004, 6:R208-R212

[14] Jacob, R.A.,Keley, D.S.,Painetto, F.S.. (1991), Immunocompetence and oxidant defense during ascorbate depletion of healthy men. Am. J Clin. Nutr. 53, 194-200.

[15] Tiku ML, Shah R, Allison GT. Evidence linking chondrocyte lipid peroxidation to cartilage matrix protein degradation. Possible role in cartilage aging and thepathogenesis of osteoarthritis. J Biol Chem. 2000 Jun; 275(26):20069-76. 
[16] Schwartz ER. Effect of vitamins C and E on sulfated proteoglycan metabolism and sulfatase and phosphatase activities in organ cultures of human cartilage. Calcif TissueInt. 1979 Nov; 28(3):201-8.

Table 1: Independent $t$-test for plasma vitamin $\mathrm{C}$ between the cases and controls.

\begin{tabular}{|l|l|}
\hline & $\begin{array}{l}\text { PLASMA VITAMIN C } \\
\text { mean+_S.D } \\
\mathrm{mg} / \mathrm{dl}\end{array}$ \\
\hline Cases & $0.7595 \pm 0.13769$ \\
\hline Controls & $1.0704 \pm 0.15926$ \\
\hline P values & $<0.001$ \\
\hline$t$ value & -14.505 \\
\hline
\end{tabular}

Table 2: Knee joint space width and plasma vitamin $\mathrm{c}$ of the study population according to the Kellgren-

Lawrence grading scale

\begin{tabular}{|l|l|l|l|l|l|l|l|l|}
\hline & GRADE 1 & \multicolumn{2}{l|}{ GRADE 2 } & GRADE 3 & \multicolumn{2}{l|}{ GRADE 4 } \\
\hline Width & Mean & 4.062 & Mean & 3.125 & Mean & 2.47 & Mean & 1.55 \\
of knee & S.D & 0.696 & S.D & 0.679 & S.D & 0.915 & S.D & 0.4972 \\
joint & Maximum & 5.5 & Maximum & 4.5 & Maximum & 4.00 & Maximum & 1.00 \\
& Minimum & 3.0 & Minimum & 2.0 & Minimum & 1.00 & Minimum & 2.5 \\
& range & 2.5 & range & 2.5 & range & 3.0 & Range & 1.5 \\
\hline Plasma & Mean & 0.881 & Mean & 0.79 & Mean & .6534 & Mean & 0.5856 \\
vitamin & S.D & 7 & S.D & 0.075 & S.D & 0.043 & S.D & 0.041 \\
C & Maximum & 0.105 & Maximum & 0.96 & Maximum & 0.73 & Maximum & 0.64 \\
& Minimum & 1.1 & Minimum & .07 & Minimum & 0.57 & Minimum & 052 \\
& range & 0.70 & range & 0.26 & range & 0.16 & Range & 0.12 \\
& & 0.40 & & & & & & \\
\hline
\end{tabular}

Table 3: Bivariate correlations between radiological grading and knee joint space width

\begin{tabular}{|l|l|}
\hline Knee joint space width & Radiological grading \\
\hline Pearson's correlation coefficient & -0.761 \\
\hline P value & $<0.001$ \\
\hline
\end{tabular}

Table 4: ANOVA of the knee joint space width and plasma vitamin $\mathrm{c}$ as per the Kellgren -Lawrence grading scale

\begin{tabular}{|cc|c|c|c|c|c|}
\hline & & Sum of Squares & df & Mean Square & F & Sig. \\
\hline Vitami & Between Groups & .880 & 3 & .293 & 48.825 & .000 \\
$\mathrm{n} \mathrm{C}$ & Within Groups & .432 & 72 & .006 & & \\
& Total & 1.312 & 75 & & & \\
\hline \multirow{2}{*}{ Width } & Between Groups & 57.846 & 3 & 19.282 & 38.842 & .000 \\
& Within Groups & 35.742 & 72 & .496 & & \\
& Total & 93.589 & 75 & & & \\
\hline
\end{tabular}

Table 5: Bivariate correlation analysis between knee joint space width, radiological grading and plasma vitamin

\begin{tabular}{|l|l|l|}
\hline Variable & Pearson's correlation coefficient $\mathrm{r}$ & $\mathrm{P}$ value \\
\hline $\mathrm{R} 1,2$ & 0.801 & $<0.001$ \\
\hline $\mathrm{R} 1,3$ & -.813 & $<0.001$ \\
\hline
\end{tabular}

X 1=plasma vitamin C, X2= knee joint space width, X3=radiological grading and R1, 2 Bivariate correlation between X1and X2, R1, 3 Bivariate correlation between X1and X3. 


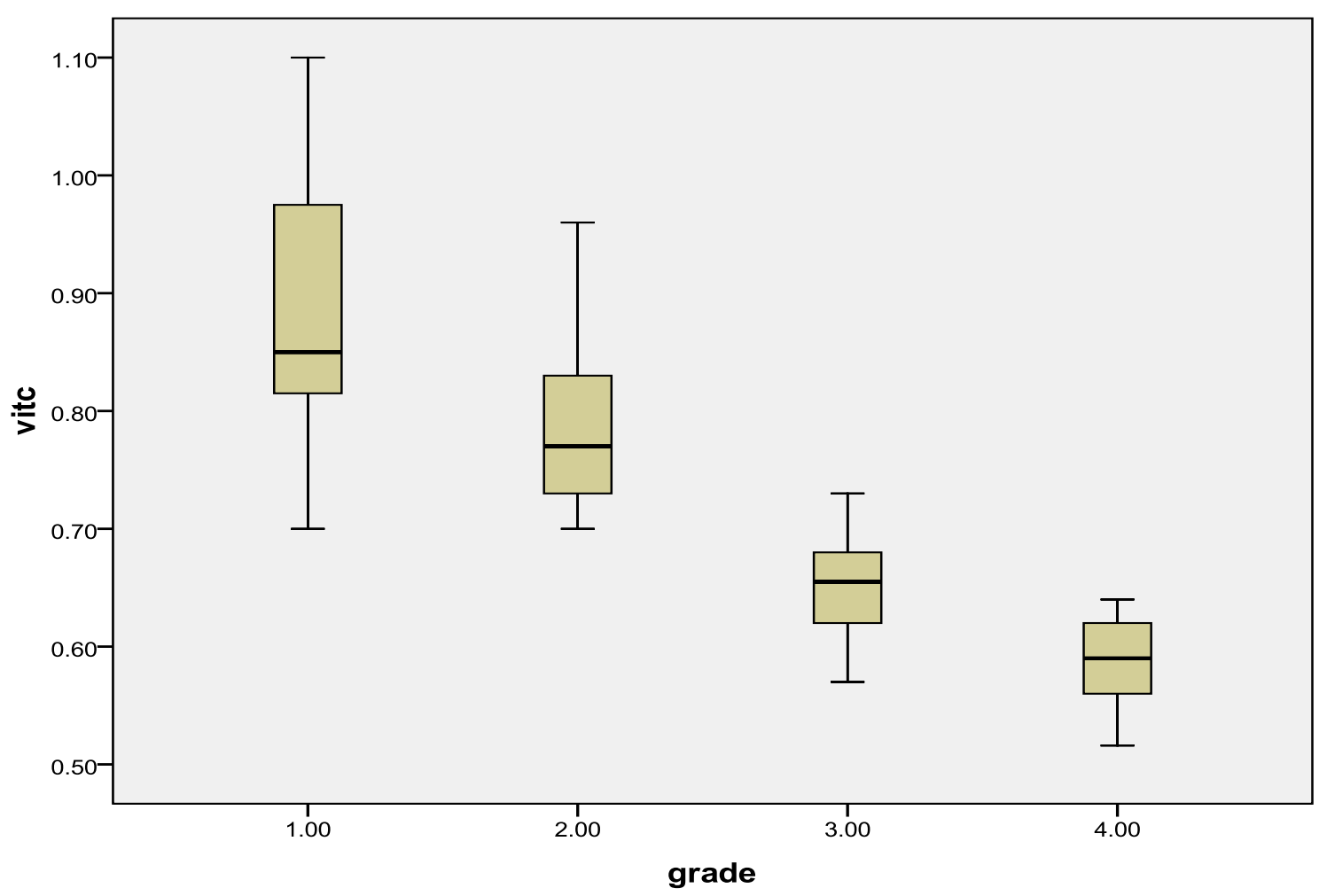

Fig.1. Box-whisker plot showing distribution of PLASMA vitamin C and Kellgren-Lawrence grade The horizontal bars inside the boxes signify median values, the limits of the boxes denote the 25th and 75th percentiles and the upper and lower whiskers .represent the range. 